Journal of Fundamental and Applied Sciences

ISSN 1112-9867

Available online at

http://www.jfas.info

\title{
SITUATION OF FLUORIDES RATE IN WATERS AND MAJOR CONSUMED FOOD IN WILAYA EL-OUED
}

\author{
A. Zobeidi ${ }^{*}$ and A. Messiatfa \\ Laboratoire de Valorisation et Promotion des Ressources Sahariennes, Université Kasdi \\ Merbah Ouargla. BP 511 route de Ghardaia, 3000 Ouargla, Algérie
}

Received: 18 November 2010 / Accepted: 06 December 2010 / Published online: 31 December 2010

\begin{abstract}
The effects of mineral salts in the drinking water, beneficial or harmful depending on their concentration, are known for many years and thus the problem posed by fluoride ions in the waters of the region (Fluorosis Dental and skeletal). This study proposes, the estimated daily intake of fluoride from its water distribution and the main food consumed per capita in the region of El-Oued (dates, tea, couscous, lentils, carrots, potatoes, pumpkin ...), which has a significant content of fluoride in their composition. In addition to the arid climatic conditions, the air temperature is very high in summer, leads to a strong human perspiration. This preliminary result leads us to propose a standard fluoride-specific region of El-Oued. The results showed that the majority of water samples and analyzed the main foods of the region of El-Oued is charged fluoride ions and exceed the maximum dose recommended for adults from 0.05 to $0.07 \mathrm{mg} / \mathrm{kg} /$ day.
\end{abstract}

Keywords: Water, Food, El-Oued, fluoride, chemical balance.

\section{INTRODUCTION}

Un accroissement considérable de population, la demande en eau potable de bonne qualité, est de plus en plus forte. En effet, les besoins en eau de l'industrie et de l'agriculture sont de plus en plus élevés.

Author Correspondence, e-mail: zobeidi.aa@gmail.com

ICID: 1020813 
Pour satisfaire cette demande, on doit recourir aux eaux sous diverses origines : superficielles et souterraines, dans certains cas, ces eaux n'est pas directement admis pour humaine à cause des concentrations en certains ions dépassant les normes de l'OMS relatives à la qualité d'eau potables.

Les effets des nombreuses substances minérales à l'organisme comme, Le $\mathrm{Ca}^{2+}$, le $\mathrm{Mg}^{2+}$, le $\mathrm{Na}^{+}$, le $\mathrm{Cl}^{-}$, le $\mathrm{F}^{-}, \ldots$. etc, contenus dans ces eaux jouent un rôle bénéfiques ou néfastes en fonction de leur concentration $[1,2]$.

En Algérie, le problème de la fluorose, dentaire et squelettique, liés à la présence des ions fluorure en teneur dépassant la norme recommandée par l'OMS (1,5 mg/l), touche de nombreuses régions du Sahara septentrional. Selon l'Association pour la Promotion de la Santé Bucco-dentaire en milieu scolaire, classe la wilaya d'EL-Oued comme zone endémique à la fluorose. En effet, et sur la base des résultats de l'enquête établie dans la région d'El-Oued, on montre que $73 \%$ de la population souffrent de la fluorose.

$\mathrm{Au}$ vue de la méconnaissance des sources potentielles de fluorure, Le travail à pour objectif d'évaluer la prise quotidienne en fluorure et de déterminer sa distribution dans les eaux et les principaux aliments consommés (dattes, thé, semoule, lentille, carottes, pomme de terre, citrouille) dans la région d'El-Oued, ou l'eau souterraine est l'unique source d'eau potable, les dattes comme aliment de base et le thé entant que boisson par excellence.

\section{MATERIEL ET METHODE}

\section{1. Échantillonnages des eaux :}

Dans la wilaya d'EL-OUED, l'alimentation en eau potable est assurée exclusivement par des eaux souterraines provenant des nappes du Complexe Terminal (CT) et du Continental Intercalaire (CI). La qualité chimique des eaux est du type sulfato-chloruré et fortement minéralisée [5]. Les échantillons ont été prélevés des forages de différentes nappes exploitées. Les forages retenus sont sélectionnés selon le nombre des patients atteints par la fluorose dentaire en basant sur les résultats d'enquête établie sur une masse importante des habitants de la wilaya d'EL-OUED. La figure 1 représente la situation géographique de wilaya d'El-Oued où les échantillons d'eau ont été prélevés. 


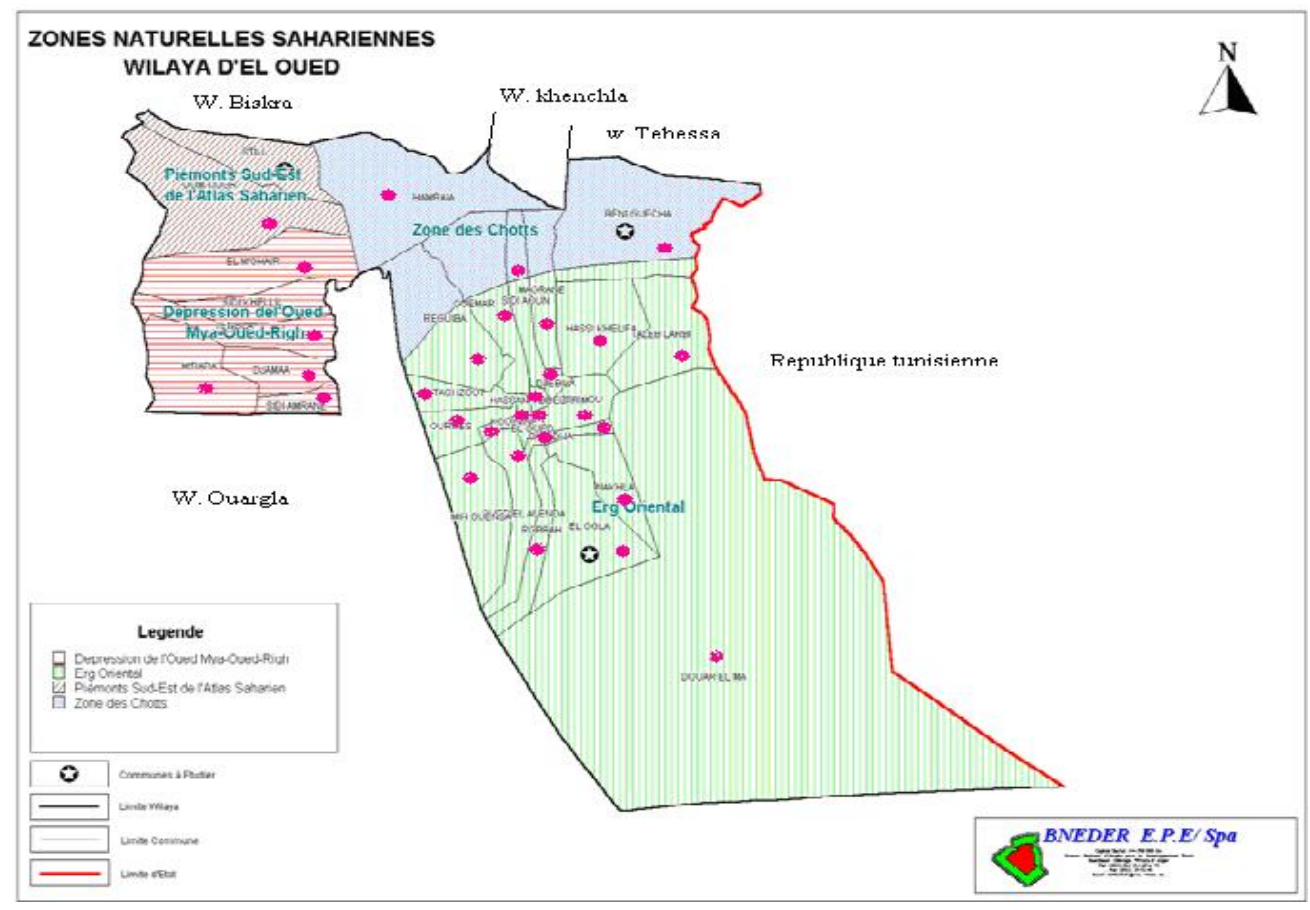

Fig.1. Commune d'El-Oued dont les eaux de forage ont été analysées.

\section{METHODES D'ANALYSE}

\subsection{Dosage des ions fluorures dans les eaux}

Une grande importance et soin ont été apportés à l'opération et méthode d'échantillonnage des eaux. Les échantillons d'eau ont été prélevés dans des flacons en plastique de $500 \mathrm{ml}$, rincés préalablement par de l'eau du forage choisi. Les paramètres

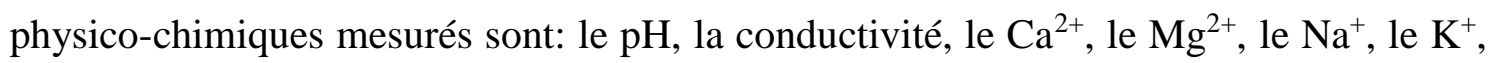
le $\mathrm{SO}_{4}{ }^{2-}$, le $\mathrm{Cl}^{-}$, les $\mathrm{NO}_{3}{ }^{-}$, et de $\mathrm{F}^{-}$. Les méthodes de dosage [6] utilisées sont les suivantes:

Le $\mathrm{pH}$ et conductivité sont mesurés respectivement, par un $\mathrm{pH}$ mètre digital, type EUTECH Instruments $510 \mathrm{pH} / \mathrm{mV} /{ }^{0} \mathrm{C}$ et par un conductimètre, type $\mathrm{HACH} 213$. Les teneurs en calcium et magnésium des échantillons sont déterminées par complexométrie, par titrage avec l'acide éthylène-diaminetétracétique (EDTA), ainsi le bicarbonate par méthode volumétrique. Les chlorures, ont été dosés selon la méthode de MOHR. La photométrie à flamme est utilisée pour doser le sodium et le potassium. Les teneurs en sulfates et en nitrates sont dosées par spectrophotométrie UV visible (type $\mathrm{HACH}$ ).

Le fluor est dosé par la méthode potentiométrique (NF T 90-004) grâce à une électrode spécifique aux ions fluorures. La mesure du potentiel a été effectuée par rapport à une 
électrode de référence au calomel $\left(\mathrm{Hg} / \mathrm{Hg}_{2} \mathrm{Cl}_{2} / \mathrm{KCl}\right.$ saturé), à l'aide d'un millivoltmètre permettant de mesurer la différence de potentiel à $0,1 \mathrm{mV}$ près. La détermination de la concentration de cet élément se fait dans des conditions expérimentales bien définies de concentrations en ions, de $\mathrm{pH}$ et de complexant. Il est à noter que l'on doit utiliser l'électrode dans une gamme de $\mathrm{pH}$ d'environ 5 à 6 pour éviter l'interférence de l'ion hydroxyde [7, 8].

D'une manière générale, il n'existe pas de normes algériennes de potabilité pour les eaux d'adduction publique. Pour cela, nous avons adopté les valeurs guide publiées par l'Organisation mondiale de la santé (OMS). La concentration admissible de ions fluorure à basse température ambiant est $1,5 \mathrm{mg} / \mathrm{l}$, mais pour une température ambiant élève ( $\mathrm{T}{ }^{\circ} \mathrm{C}>40{ }^{\circ} \mathrm{C}$ en été), induit une forte consommation d'eau, la concentration admissible 0,85 mg/l (suivant de température durant 1998-2008).[9,10].

\subsection{Teneurs des ions fluorures dans les aliments}

Le dosage des ions fluorure dans les dattes et les autres aliments consommé par les habitants des régions étudiées, ont été calcinées à $500^{\circ} \mathrm{C}$ jusqu'à la stabilité du poids du charbon obtenu. En effet, les composantes de fluorures risquent de devenir volatiles au delà de $550^{\circ} \mathrm{C}[3,11]$. Une fois refroidis, les charbons de datte sont broyés doucement jusqu'à l'obtention d'une poudre homogène. La minéralisation, consiste à la mise en contacte d'une masse de $5 \mathrm{gr}$ avec un volume d'acide nitrique concentré. Après 24 heures de réaction, le minéralisât est ajusté à $100 \mathrm{ml}$ de l'eau distillée, puis filtré soigneusement avec un filtre millipore. La filtration est répétée jusqu'à l'obtenir d'une solution claire $[3,12]$. A l'analyse les échantillons sont ajustés à un $\mathrm{pH}$ compris entre 5-6 en utilisant le TISAB.

Pour être compléter le bilan de consommation, trois variétés de thé vert les plus commercialisées dans la région est mises à l'étude pour quantifier sa teneur en fluorure. Sa concentration dépend non seulement du type de thé mais aussi de l'eau utilisée. Pour ce faire, différentes préparations de thé ont été obtenues avec de l'eau filtrée et l'eau du robinet [12].

\section{RESULTATS ET DISCUSSION}

Les prélèvements d'échantillons d'eaux souterraines ont été prélevés, entre les mois de janvier et mai 2009, sur trente (30) forages d'eaux alimentant la wilaya d'El-Oued en 
eau potable. Les caractéristiques physico-chimiques de ces eaux prélevées sont consignées dans le tableau 1. Elles représentent la moyenne de trois essais.

Les eaux sont fortement minéralisées de conductivité allant de 2,17 à $7,13 \mathrm{mS} / \mathrm{cm}$ et d'un $\mathrm{pH}$ compris entre 7 et 7,94, révèlent ainsi une légère alcalinité bicarbonatée. La teneur en magnésium est varie entre 60,76 et $211,45 \mathrm{mg} / \mathrm{l}$, soit $77 \%$ des eaux échantillonnées présentent un taux de magnésium supérieur à $100 \mathrm{mg} / \mathrm{l}$. Concernant le calcium, sa concentration varie d'un forage à l'autre de 204 à 545 mg/l. Selon les concentrations de calcium et de magnésium, ont déduit que la dureté totale est relativement élevée. D'autre part, les eaux présentent des teneurs élevées en chlorures et en sulfates varient respectivement entre 320 à $1264 \mathrm{mg} / \mathrm{l}$ pour les chlorures et de 304 à $973 \mathrm{mg} / \mathrm{l}$ pour les sulfates. Concernant les ions sodium, 77\% des eaux présentent en moyenne des teneurs supérieures à $200 \mathrm{mg} / \mathrm{l}(102$ à $589 \mathrm{mg} / \mathrm{l})$. Pour le potassium les teneurs allant de 40 à $86 \mathrm{mg} / \mathrm{l}$. Concernant les nitrates, on note des teneurs non négligeables, cependant, avec la forte profondeur des nappes et dans la faible activité industrielle et agricole, il semble que ces résultats seront attribués à la méthode analytique [3].

Pour les ions fluorures, axe principale de cette étude, on montre que $87 \%$ des eaux échantillonnées (soit, 26 forages), présentent des teneurs en fluorures supérieure à la norme de l'OMS 1,5 mg/l. Les concentrations varient d'une région à l'autre, de 0,62 à $2,63 \mathrm{mg} / \mathrm{l}$. Les fortes teneurs $(2,65 \mathrm{mg} / \mathrm{l})$ s'observent dans les eaux du forage de "Chouhada 1", captant la nappe du Complexe terminal. Cependant, les plus faibles $(0,62$ mg/l), s'observent pour les eaux due forage de " Tindla ", captant la nappe du continental Intercalaire. Cette variation de concentration en ion fluorure est attribuée à l'aspect géologique du réservoir aquifère. Les roches magmatiques, notamment, peuvent renfermer de fortes concentrations en fluorures. D'autre part, la possibilité de la libération de fluorure à partir du substrat géologique (fluorure de calcium) est un critère important. En plus, les conditions climatiques sont à prendre en considération, puisque le climat sec et aride favorise l'accumulation des sels, notamment de fluorure, dans les eaux souterraines les plus proches de la surface. Enfin, le $\mathrm{pH}$ du sol est un paramètre important puisque les fluorures sont libérés sous forme anionique en milieu basique [16]

\subsection{Dose optimal (mg/l) des ions fluorures dans l'eau}


Il est à noter, que la concentration recommandée en ions fluorures dans les eaux de consommation est une fonction de la température de l'air ambiant comme l'indique l'équation de Galgon et Vermilion [17] suivante :

\subsection{Dose optimal $(\mathrm{mg} / \mathrm{l})$ des ions fluorures dans l'eau $=0.34 /\left(-0.038+0.0062 \mathrm{~T}_{\mathrm{M}}\right)$}

Tableau 1. Variation de la concentration en ion fluorure dans les eaux de consommation en fonction de la température de la région d'El-Oued (Période 1998 à 2008)

\begin{tabular}{|c|c|c|c|c|c|c|c|c|c|c|c|c|c|}
\hline Mois & Jan. & Fév. & Mars & Avr. & Mais & Jui. & Juil. & Août & Sept. & Oct. & Nov. & Déc. & $\begin{array}{c}\text { Moy. } \\
\text { annuelle }\end{array}$ \\
\hline $\begin{array}{c}\text { Tempé } \\
\left({ }^{\mathbf{C}}\right)\end{array}$ & 10,76 & 12,92 & 17,84 & 21,56 & 26,45 & 31,3 & 34,43 & 33,83 & 28,67 & 25,33 & 16,36 & 11,65 & 22,59 \\
\hline $\begin{array}{c}F^{-} \\
(\mathbf{m g} / \mathbf{l})\end{array}$ & 1,21 & 1,11 & 0,94 & 0,84 & 0,74 & 0,65 & 0,62 & 0,63 & 0,70 & 0,76 & 0,99 & 1,71 & 0,85 \\
\hline
\end{tabular}

Pour une température moyenne annuelle de $22,59{ }^{0} \mathrm{C}$, au cours des 10 dernières années (1998-2008), la quantité de fluorure optimale pour l'eau potable de ces zones, est de $0,85 \mathrm{mg} / \mathrm{l}$. 
Tableau 2. Caractéristiques physico-chimiques des eaux souterraines de la région d'El-Oued

\begin{tabular}{|c|c|c|c|c|c|c|c|c|c|c|c|c|c|c|}
\hline Localisation & Nappe & $\mathbf{p H}$ & $\begin{array}{c}\text { Cond. } \\
\left(\mathrm{mS.cm}^{-1}\right)\end{array}$ & $\begin{array}{c}\text { TH } \\
\left(\mathrm{mg.l}^{-1}\right)\end{array}$ & $\begin{array}{c}\text { TAC } \\
{ }^{\circ} \mathbf{F}\end{array}$ & $\begin{array}{c}\mathrm{Ca}^{2+} \\
\left(\mathrm{mg.l}^{-1}\right)\end{array}$ & $\begin{array}{c}\mathrm{Mg}^{2+} \\
\left(\mathrm{mg.l}^{-1}\right)\end{array}$ & $\begin{array}{c}\mathrm{Na}^{+} \\
\left(\mathrm{mg.l}^{-1}\right)\end{array}$ & $\begin{array}{c}\mathbf{K}^{+} \\
\left(\mathbf{m g . l ^ { - 1 }}\right)\end{array}$ & $\begin{array}{c}\mathrm{HCO}_{3}^{-} \\
\left(\mathrm{mg.l^{-1 }}\right)\end{array}$ & $\begin{array}{c}\mathrm{Cl}^{-} \\
\left(\mathrm{mg.l^{-1 }}\right)\end{array}$ & $\begin{array}{c}\mathrm{SO}_{4}^{-} \\
\left(\mathrm{mg.l}^{-1}\right)\end{array}$ & $\begin{array}{c}\mathrm{NO}_{3-} \\
\left(\mathrm{mg.l}^{-1}\right)\end{array}$ & $\begin{array}{c}\cdot \\
\left(\mathrm{mg} . \mathrm{I}^{-1}\right) \\
\end{array}$ \\
\hline Debila & Mio-plio & 7,57 & 3,66 & 1590 & 12,4 & 440,88 & 119,09 & 440,33 & 70,40 & 151,28 & 801,23 & 510 & 7,1 & 2,25 \\
\hline Hassani & Mio-plio & 7,41 & 3,50 & 1190 & 11,4 & 296,59 & 109,37 & 470,13 & 77,40 & 139,08 & 893,16 & 533 & 6,3 & 2,07 \\
\hline Oued El Alenda & Mio-plio & 7,50 & 1,75 & 1390 & 13,3 & 296,59 & 157,98 & 460,33 & 82,07 & 162,26 & 198,08 & 492 & 4,9 & 1,89 \\
\hline Sidi kahelli & Mio-plio & 7,20 & 7,13 & 2490 & 11,4 & 545,08 & 174,64 & 379,04 & 55,75 & 139,08 & 1264,21 & 806 & 8,4 & 2,64 \\
\hline El Robah & Mio-plio & 7,61 & 3,47 & 1100 & 13,1 & 264,53 & 109,37 & 460,33 & 82,07 & 161,04 & 815,42 & 468 & 4,9 & 1,91 \\
\hline El Nakhla & Mio-plio & 7,43 & 3,46 & 1050 & 13,1 & 256,51 & 99,65 & 450,53 & 81,68 & 159,82 & 801,23 & 304 & 9,4 & 1,86 \\
\hline Sidi Aoun & Mio-plio & 7,48 & 3,51 & 1190 & 12,7 & 276,55 & 121,62 & 440,73 & 72,74 & 154,94 & 815,41 & 442 & 6,5 & 2,10 \\
\hline El Ogla & Mio-plio & 7,10 & 3,51 & 1090 & 13,1 & 272,54 & 99,65 & 440,73 & 85,96 & 159,82 & 850,85 & 710 & 5,0 & 2,03 \\
\hline Hassani /Karim & Mio-plio & 7,42 & 3,41 & 1100 & 11,5 & 272,55 & 102,08 & 430,92 & 73,52 & 140,30 & 894,14 & 710 & 5,0 & 2,20 \\
\hline Kouininne & Pontien & 7,30 & 4,08 & 1390 & 12,9 & 296,59 & 157,98 & 432,90 & 44,50 & 157,38 & 953,68 & 573 & 6,2 & 2,07 \\
\hline Chouhada 1 & Pontien & 7,28 & 4,34 & 1318 & 13,1 & 312,62 & 155,54 & 470,14 & 75,46 & 159,82 & 829,60 & 544 & 5,9 & 2,63 \\
\hline El Hamraïa & Pontien & 7,26 & 3,44 & 1330 & 12,8 & 316,63 & 150,69 & 470,14 & 57,57 & 156,16 & 836,69 & 510 & 4,9 & 1,73 \\
\hline El Meghaier & Pontien & 7,84 & 3,45 & 1230 & 12,8 & 392,78 & 60,76 & 180,96 & 45,45 & 156,16 & 758,69 & 694 & 5,8 & 2,05 \\
\hline Still & Pontien & 7,41 & 4,83 & 1690 & 13,0 & 355,70 & 192,57 & 181,96 & 49,86 & 158,60 & 935,95 & 512 & 6,0 & 2,13 \\
\hline El- oued & Pontien & 7,55 & 4,77 & 1390 & 14,5 & 288,58 & 162,84 & 116,22 & 58,69 & 176,90 & 829,60 & 546 & 5,1 & 10 \\
\hline Mouih Ouensa & Pontien & 7,23 & 4,76 & 1390 & 13,9 & 296,59 & 157,98 & 392,76 & 60,23 & 169,58 & 699,23 & 400 & 5,1 & 1,94 \\
\hline Hassi Kahlifa & Pontien & 7,83 & 3,58 & 1360 & 13,3 & 384,76 & 97,22 & 146,49 & 60,16 & 162,26 & 794,14 & 712 & 7,1 & 2,17 \\
\hline Reguiba & Pontien & 7,94 & 4,85 & 1030 & 13,0 & 308,10 & 161,38 & 589,03 & 39,57 & 158,60 & 815,41 & 572 & 6,3 & 2,04 \\
\hline Ourmes & Pontien & 7,66 & 5,14 & 1590 & 12,7 & 348,69 & 174,99 & 203,86 & 58,69 & 154,94 & 1006,8 & 533 & 8,1 & 2,09 \\
\hline Guemmar & Pontien & 7,39 & 4,92 & 1630 & 13,8 & 308,61 & 211,45 & 505,82 & 48,39 & 168,36 & 872,14 & 585 & 6,1 & 2,19 \\
\hline Trifaoui & Pontien & 7,72 & 3,44 & 1590 & 13,4 & 384,76 & 150,69 & 411,31 & 72,57 & 163,48 & 801,23 & 698 & 5,9 & 2,17 \\
\hline El Bayada & Pontien & 7,73 & 3,46 & 1060 & 12,7 & 268,54 & 94,78 & 450,53 & 79,35 & 154,94 & 815,41 & 469 & 7,4 & 2,01 \\
\hline El Magrane & Pontien & 7,39 & 3,45 & 1230 & 12,7 & 392,78 & 60,76 & 430,92 & 72,35 & 154,94 & 758,69 & 442 & 5,8 & 2,02 \\
\hline Guemmar & Pontien & 7,47 & 4,87 & 1660 & 13,2 & 352,70 & 189,57 & 181,96 & 51,33 & 161,04 & 935,95 & 507 & 6,3 & 2,18 \\
\hline Benguecha & Eocène & 7,80 & 5,66 & 1100 & 11,6 & 360,72 & 149,28 & 203,86 & 43,98 & 141,52 & 893,41 & 559 & 4,1 & 2,08 \\
\hline Sidi Amran & Albien & 7,51 & 2,77 & 1050 & 16,3 & 252,50 & 104,51 & 102,00 & 73,39 & 198,86 & 397,03 & 570 & 1,8 & 0,85 \\
\hline Djamaa & Albien & 7,24 & 2,76 & 1030 & 15,0 & 204,40 & 126,38 & 212,18 & 52,80 & 183,00 & 404,16 & 620 & 1,5 & 0,67 \\
\hline Tindla & Albien & 7,49 & 2,74 & 1180 & 14,2 & 236,47 & 143,39 & 212,18 & 60,16 & 173,24 & 389,98 & 530 & 1,7 & 0,62 \\
\hline Chouhada II & Barrémien & 7,03 & 2,17 & 1100 & 14,1 & 368,75 & 97,22 & 283,86 & 57,80 & 172,02 & 617,99 & 973 & 1,8 & 0,63 \\
\hline Hassi Kahlifa & Barrémien & 7,01 & 2,31 & 810 & 13,8 & 348,64 & 191,3 & 146,49 & 60,16 & 168,36 & 876,3 & 952 & 4,3 & 0,67 \\
\hline
\end{tabular}




\subsection{Distribution des ions fluorures dans les principaux aliments consommés}

La teneur en fluorure contenue dans les différentes variétés des dattes, varie de 30,8 à 74,5 et $\mathrm{mg} / \mathrm{kg}$, témoins naturels prélevés des sols et des eaux comme le montre les travaux de $[3,12]$. Les plus fortes teneurs s'observent pour la variété Daglet noor $(74,5 \mathrm{mg} / \mathrm{kg})$.

La concentration de fluorure varie selon le type des feuilles de thé utilisées et du mode de préparation. Elle est de 1,82 à 2,88 mg/l en première préparation, de 1,21 à 1,95 mg/l en deuxième préparation et de 0,74 à $1,44 \mathrm{mg} / \mathrm{l}$ en troisième préparation, soit un apport total journalier de 3,79 à $6,27 \mathrm{mg} / \mathrm{l}$. Ces résultats montrent que le thé présente un apport important d'ion fluorures pour les consommateurs.

Les autres principaux aliments consommés dans la région, ont des teneurs variables. Les teneurs les plus élevées $(44 \mathrm{mg} / \mathrm{kg}$ ) ont été dosées dans les sels et la plus faible est enregistrée pour les tomates $(3,8 \mathrm{mg} / \mathrm{kg})$.

\subsection{Bilans de masse de l'ion fluorure consommé}

Il est pratiquement difficile de déterminer l'apport total en ion fluorure consommé par jour et par habitant de la région d'EL-Oued. Le fluorure est pratiquement présent dans tous les aliments. Les légumes [11,13], les dattes [1], thé [3,11,14,15], le sel [11], les fruits [15],sont les aliments les plus riches en ion fluorure.

La quantité du fluorure totale journalière ingérée par un adulte habitant cette région est estimée selon l'équation de [13].

$$
\text { La consommation journalière totale }=\sum_{i} C_{j} I_{i}
$$

Tel que: i, représente la source (eau, thé, datte, et autres aliments), $\mathrm{C}$, la concentration de la source (g/jour ou 1/jour), I, la consommation des sources (g/jour ou 1/jour).

Les résultats obtenus montre que la quantité de fluorure administrée liée a la consommation d'eau varie selon la source (forage), de 0,62 à 2,63 mg/l, soit en moyenne $3,10 \mathrm{mg} / 1,91$ d'eau consommée par jour. Pour un homme qui consomme 0,2 1/jour de thé, l'apport du fluorure lié au thé varie de 3,56 à 5,27 mg/l, soit en moyenne de 0,88 $\mathrm{mg} / 0,21$. Les dattes apports, selon la variété, entre 30,84 et $74,48 \mathrm{mg} / \mathrm{kg}$, soit en moyenne de $5,26 \mathrm{mg} / 0,1 \mathrm{~kg}$ de datte consommé et pour les autres aliments, la quantité consommée est de $8,13 \mathrm{mg} / 0,34 \mathrm{~kg}$. Ainsi, la quantité total de fluorure consommé par un adulte habitant cette région est de 19,4 mg/jour. Pour un habitant adulte de $60 \mathrm{~kg}$, la dose ingérée est de $0,29 \mathrm{mg} / \mathrm{jour} / \mathrm{kg}$; soit 5 fois supérieure à la norme recommandée pour un adulte $(0,05$ à $0,07 \mathrm{mg} / \mathrm{jour} / \mathrm{kg})[18]$. 


\section{CONCLUSION}

Les résultats ont montré que les principaux sources de fluor sont: l'eau de 0.62 à 2.64 $\mathrm{mg} / \mathrm{l}$, et $87 \%$ qui dépassent les normes recommandés par l'OMS $(0.85 \mathrm{mg} / \mathrm{l}$ suivant la température). Pour le thé, la teneur dépend de la durée d'infusion des feuilles et la quantité de thé, elle est de 4 à $6 \mathrm{mg} / \mathrm{l}$. Par ailleurs, la teneur en fluorure contenue dans les différents variétés des dattes varie entre 30,84 à 74,48 mg/l. les autres alimentes les plus consommées dans la région, Les teneurs les plus élevées $(44 \mathrm{mg} / \mathrm{kg})$ ont été dosées dans les sels et la plus faible est enregistrée pour les tomates $(3,8 \mathrm{mg} / \mathrm{kg})$. Ainsi, la quantité total de fluorure consommé par un adulte habitant cette région est de 19,4 $\mathrm{mg}$ /jour. Pour un poids corporelle moyen de $60 \mathrm{~kg}$ d'un adulte habitant la région d'étude, la dose journalière consommée, dépasse l'optimale recommandée de 0,05à 0,07 $\mathrm{mg} / \mathrm{kg} /$ jour nécessaire ainsi une défluoruration des eaux ou une diminution de la consommation des aliments riches en fluorure.

\section{REFERENCES}

[1] Viland M., Montiel A. 2001, Guide pratique pour les intervenants en milieu rural africain, Eau et Santé, Programme Solidarité Eau, Editions du Gret, France.

[2] Behrman A. S., Gustafson H. Ind. Eng. Chem. 1938, $30,9$.

[3] Messaitfa A. Environ Geol. 2008, 55, 377-383.

[4 ] Bannoud A., Darwich Y. Desalination.2007, 206 ,449-456.

[5] Djellouli H.M., Taleb S., Harra D. Cahiers d'études et de recherches francophones/santé. 2005, 15 (2), 109-112.

[6] Rodier J. 2005, L'analyse de l'eau: eaux naturelles, eaux résiduaires, eaux de mer, 8e éd, Paris Bordas, 1345 p.

[7] Ferry D., Machtinger M., Bauer D. Analysais, 1984, 2, 90-5.

[8 ] NF T 90-004. Essais des eaux : dosage de l'ion fluorure : méthode potentiométrique Septembre 1985.

[9] Organisation mondiale de la santé (OMS).Directives de qualité pour l'eau de boisson. Recommendations. Vol. 1. 2e éd. Genève: OMS, 1994, 187-95.

[10] World Health Organization (WHO). Guidelines for Drinking-water Quality. Chemical summary tables. Vol. 1, 3rd ed. Appendix 4. Geneva : WHO, 2004, 488-93

[11] Alex H., Sri S., Manfred van B., Budi W., Luuk F. Science of the Total Environment. 2005, 346, 56-69. 
[12] Lachouri A.(2009). Distribution des ions fluorures dans les eaux et les principaux alimentes. Evaluation du risque de la fluorose dans deux communautés du Sud-Est Algérien (Ouargla et El-Oued). Thèse de Magister, Université de Ouargla.

[13] Heikens A., Sumartib S., Bergenb M., Widianarkod B., Fokkerte L., Leeuwenf K., Seinena W. Science of the Total Environment. 2005, 346, 56-69.

[14] Yi J., Cao J. Journal of Fluorine Chemistry. 2008, 129, 76-81.

[15] Danrong S., Maixime P., Jean-Christophe S., Let Courfia A ., Diawara K. Elimination domestique et industrielle du fluor en excès, 2009, $\mathrm{N}^{\circ}$ 301-302.

[16] Lhassani A. Rumeau M., Benjelloum D., Pontie M. Water Res. 2001, 35, 3260.

[17] Galgon D. J., Vermilion J. R. Public health rep. 1957, 72, 484-490.

[18] Levy S. S., Ambik S. R. et Prasao S. J, Review of fluoride contamination Dent Oral Epidemiol, 1994, 173p. 


\section{SITUATION DU TAUX FLUORURES DANS LES EAUX ET LES PRINCIPAUX ALIMENTS CONSOMMES DANS LA WILLAYA EL-OUED}

\section{RESUME}

Les effets des sels minéraux contenus dans les eaux de boisson, bénéfiques ou néfastes en fonction de leur concentration, sont connus depuis de nombreuses années et partant de la problématique posée par les ions fluorure dans les eaux de la région (Fluorose dentaire et squelettique). Cette étude se propose, d'évalué la prise quotidienne en fluorure à partir de sa distribution dans les eaux et les principaux aliments consommés par habitant de la région d'El-Oued (dattes, thé, semoule, lentille, carottes, pomme de terre, citrouille...), dont le fluorure présente une teneur significative dans leur composition. En outre les conditions climatiques arides ou' la température de l'air est très élevée en été, entraîne une forte transpiration du corps humaine. Ce résultat préliminaire, nous mène à proposer une norme de fluorure propre à la région d'ElOued.

Les résultats obtenus ont permis de constater que la majorité des échantillons d'eau et les principaux aliments analysés de la région d'El-Oued sont chargés des ions fluorures et dépassent la dose l'optimale recommandée pour un adulte $0,05 a ̀$ 0,07 mg/kg/jour.

Mots clés : L'eau potable, Aliments, El-Oued, Fluorure, Bilan chimique.

\section{How to cite this article}

Zobeidi A and Messiatfa A. Situation of fluorides rate in waters and major consumed food in wilaya El-Oued. J Fundam Appl Sci. 2010, 2(2), 302-312. 